\title{
High-Frequency Trading and Its Impact on Exogenous Liquidity Risk of China's Stock Index Futures Market before and after Trading Restrictions
}

\author{
GuangWei Shi ${ }^{1,2}$ and Yun Chen (D) \\ ${ }^{1}$ Shanghai University of Finance and Economics, Shanghai, China \\ ${ }^{2}$ Shanghai Financial Futures Information Technology Co., Ltd., Shanghai, China \\ Correspondence should be addressed to Yun Chen; chenyun@sufe.edu.cn
}

Received 14 May 2020; Accepted 30 June 2020; Published 27 August 2020

Guest Editor: Benjamin Miranda Tabak

Copyright (C) 2020 GuangWei Shi and Yun Chen. This is an open access article distributed under the Creative Commons Attribution License, which permits unrestricted use, distribution, and reproduction in any medium, provided the original work is properly cited.

\begin{abstract}
Since China's first stock index futures, China Securities Index 300 (CSI300) stock index futures were published in 2010, and China's stock index futures market is now in a period of rapid development and play a key role in price discovery. During 2014 to 2015, China's stock index futures market fluctuated abnormally, and the overuse of high-frequency trading (HFT) strategies in the stock index futures market was blamed as the main reason of the abnormal volatility. To lower down market fluctuation, the regulatory institute then announced a series of trade restriction policy to prevent the overuse of HFT behaviour. However, until now, the impact of such trade restriction policy for HFT remains uncertain. To tackle this issue, based on minute-level HFT data from the CSI 300 index futures market, this paper aims to investigate the relationship between HFT and the exogenous liquidity risk and how HFT affects China's stock index futures market on its liquidity using the liquidity-adjusted value at risk (LVaR) model. The findings indicate that HFT improves the return of the liquidity provider and reduces the exogenous liquidity risk significantly.
\end{abstract}

\section{Introduction}

Stock index futures are an important financial derivative, and their main function is to maintain capital values and avoid systematic risk in the stock market. Since the stock index futures market is closely related to its stock spot market, coupled with the principle of leveraged trading of futures, the marginal risk of the stock index futures market may cause risks in the spot market through the risk transmission mechanism. Nowadays, with the rapid development of trading technology in recent years, high-frequency trading, a kind of programmatic trading, based on low latency between order submission and execution or cancellation, is booming in the China stock index futures market. However, HFT may make bid-ask spread in low level, and hence it could easily cause liquidity risk and pose new challenges to the risk management of the China stock index futures market. In this circumstance, investors may bear huge liquidity risk leading to huge losses, and the market will lose its function [1].

On June 12, 2015, the Shanghai Stock Exchange Composite Index ( $\mathrm{SH}$ index) reached 5178, a new peak in recent years. After that, the spot market fluctuated violently, over a thousand stocks went through stock suspension during that period, trading volume dropped sharply, and market liquidity hit the freezing point. Some scholars think that the overuse of HFT in the stock index futures market is the key factor of the sharp fluctuation of the spot market. From August to September in 2015, the China Financial Futures Exchange (CFFEX) issued a series of stock index futures trading restriction measures to lower the sharp fluctuations of the stock market and reduce the excessive speculation behaviour in the market. The policies aim to regulate the trading behaviour of the stock index futures market through 
lifting the HFT cost in order to promote the healthy development of the China stock index futures market. However, it is reported that these regulatory measures such as increasing margin and limiting opening positions have a series of negative effects on the futures market and the spot market, respectively, especially for the liquidity level on both markets. Liquidity refers to the possibility that investors can trade a certain asset with less trading time, lower transaction cost, reasonable price level, and smaller price fluctuation, while liquidity risk means all kinds of risks such as sharp rise in transaction costs, extended trading time, and difficulty in realizing due to the loss of investors' confidence. Lack of liquidity in the market may make it hard for investors to complete the transaction.

In the existing research studies on liquidity risk, many researchers have expounded the importance of the liquidity risk. Jorion [2] pointed out that liquidity is the most important issue in risk management. The vast majority of unforeseeable losses are caused by the sudden disappearance of liquidity due to the lack of liquidity in the market or by increased clearing costs due to the market moving in the opposite direction to the traders' positions. Amihud and Mendelson [3] described liquidity as "everything in the market." Lawrence and Robinson [4] mentioned that ignoring liquidity risk is much likely to lead to undervaluation of the market risk, which could be about 15 percent. Dowd [5] thought that losses caused by liquidity risks may even be as large as those caused by market risks. Pastor and Stambaugh [6] proved in their empirical study that the market shock cost in the direction of buying or selling is used as the state variable to measure the market liquidity risk.

Because of the characteristics of the liquidity risk as an indispensable systemic risk and its important position in risk management, researchers have been trying to conduct indepth research on liquidity risk for decades, in order to find a more suitable liquidity risk measurement model for the capital market, so as to better understand the market where they are located. As to the measurement of the liquidity risk, Bangia, Diebold, and Stroughair [7] divide liquidity into exogenous and endogenous components.

Exogenous liquidity is determined by market characteristics and has the same impact on each market participant, independent of the individual trading behaviour of individual market participants [8]. Markets with good exogenous liquidity tend to have much trading volume and more stable bid-ask spread. On the contrary, bid-ask spread in the market with poor exogenous liquidity fluctuates greatly, while the depth of declaration and trading volume is relatively small. Endogenous liquidity is related to the positions held by investors. Generally speaking, the larger the positions held by investors, the worse the endogenous liquidity. However, the measurement of endogenous liquidity needs to be based on the assumption of positions, and the data of investors' positions are often not easy to obtain. Therefore, the computation in this paper takes only the risk of exogenous liquidity that will affect any investor into account and ignores the possible impact of endogenous liquidity.

With the development of financial theory and financial engineering, financial market risk measurement technology has become more comprehensive. At present, the main methods of financial market risk measurement include the sensitivity analysis method, volatility analysis method, VaR method [2], stress test [9], extreme value theory [10], and Copula [11]. Among the above methods, the value at risk model (VaR) is one of the mainstream methods on risk measurement in the financial market. Since the 1990s, it has been introduced into risk management and gradually widely used in the field of market risk measurement and supervision. However, the traditional VaR model still has some limitations. For instance, traditional VaR models do not take the impact of investors' trading behaviors on market prices (or market shocks) into account. The traditional VaR model is based on the assumption that investors can clear in a short time, so it ignores the possible losses caused by price fluctuations. Furthermore, the impact of clearing on bid-ask spreads in market makers' markets is also not taken into account.

However, the VaR model ignores that the big deals may lead to market price and price fluctuation; in order to improve the deficiency of the VaR model, Hisata and Yamai [12] argure that the uncertainty of bid-ask spread can be regarded as an indicator of liquidity risk, so the volatility of bid-ask spread is introduced into exogenous cost of liquidity (ECL), and they propose the LVaR model (liquidity-adjusted value at risk). As for domestic research studies in China on the liquidity risk study, $\mathrm{Hu}$ and Song [13] took the stock price of 16 listed commercial banks in China as samples to establish the VaR model after liquidity risk adjustment. Based on the day-level trading data of Shanghai stock exchange, Zhu [14] defined the measure index of liquidity risk from the perspective of the price shock faced by investors in actual investment. Xu et al. [15] used the VaR model to verify the liquidity risks of enterprises with different liquidity in the NEEQ (National Equities Exchange and Quotations) market under different market conditions and proposed the corresponding regulation policy. Tong et al. [16] theoretically analyzed daylevel liquidity risk, studied the relationship between regulatory indexes and day-level liquidity risk, and explored the deficiencies of regulatory framework indexes.

1.1. Highlights. Despite considerable research results on liquidity risk, they are mainly concentrated in the spot market such as the stock market. At present, due to the complexity of the liquidity risk formation mechanism in the futures market, relevant research on HFT in China's stock index futures market is still scarce, and systematic research work has not yet been carried out, not to speak at intraday level. The above detail illustrates the importance of liquidity risk measurement of the stock index futures market and the lack of relevant theoretical research in China. Given the importance of that, research on the stock index futures market in China based on the financial market microstructure theory is carried out in this paper. Specifically, the LVaR model is used to quantify the liquidity risk using minute-level HFT data of CSI 300 index futures.

The main contribution of this study includes the following: 
(1) The experimental data used in this paper are based on minute-level HFT data ranging from 2014 to 2016 which spans the entire stock index futures market turbulent period containing the period under trading restrictions, which is beneficial to systematically study the relationship between HFT and liquidity risk in the stock index futures market, and has an extremely valuable research value.

(2) The liquidity risk model is illustrated by LVaR to demonstrate how HFT affects liquidity on the CSI 300 index futures market. The research results and conclusions are of great significance to the market risk management of other financial futures, such as providing reference for the risk monitoring of other stock index futures products, treasury futures products, and other financial derivatives that may be launched in the future.

The rest of the paper is organized as follows. Section 2 briefly describes the problem statement including the basic concepts of liquidity risk and why the specific range of data is selected for the experiment. In Section 3, the relationship between HFT and liquidity is demonstrated by the VAR model. In Section 4, the LVaR model is introduced to model exogenous liquidity risk, and the impact of HFT is deeply analyzed. Section 5 concludes the paper.

\section{Problem Statement}

Regarding if HFT is beneficial to the liquidity risk in the stock index futures or not, the research community has been controversial. Besides, to the best of our knowledge, the impact of HFT on China's stock index futures market has not been deeply analyzed before. In this context, the study of HFT on China's stock index futures market has both theoretical and practical meanings.

In this section, the definition of liquidity risk is first introduced. And, the range of experimental data used in this paper and why we choose that to demonstrate the liquidity risk brought by HFT are then presented.

2.1. Concepts of Liquidity. Liquidity was first proposed by Keynes in 1936 and is now broadly defined as "liquidity is a price-balanced ability to buy and sell large amounts of certain financial assets without fluctuating their prices."

Bangia et al. [7] think liquidity should be classified into two parts: endogenous liquidity and exogenous liquidity. Exogenous liquidity is determined by market characteristics and is not affected by the individual trading behaviour of individual market participants and is the same for each market participant.

The market trade volume with good exogenous liquidity is very large, the bid-ask spread is small and stable, and the quotation depth (the possible trading volume under the existing bid-ask price level of the market maker) is high. In this case, the realization cost is relatively small and can even be ignored. Conversely, a market with poor exogenous liquidity would be characterized by a large fluctuation in bid-ask spreads and a very small quotation depth and trading volume.

As can be seen from Figure 1, the relationship between position size and liquidation value is as follows: when the quantity of the buy order (sold) is less than the depth of the buy order, the transaction can be traded at the current quotation level. At this time, only the exogenous liquidity risk exists, and there is no endogenous liquidity risk. On the contrary, when the amount of buying (selling) is higher than the quotation depth, the realized cost of the asset will exceed the current quotation level, which will cause the exogenous liquidity risk and the endogenous liquidity risk to exist at the same time. The portion of the cost is reflected as the endogenous liquidity risk.

Endogenous liquidity, on the other hand, is related to positions held by market participants. Generally speaking, the larger the position held by market participants, the worse the endogenous liquidity. Figure 1 shows the relationship between position and the realized value. The measurement of endogenous liquidity needs to be based on the assumption of the position volume, which is not available. Therefore, in the following liquidity risk calculation, only the risk brought by the exogenous liquidity is considered.

\subsection{Trade Regulation Change and Its Impact on CSI 300 Index} Futures. Since the CSI 300 index future was published in April in 2010, the trade regulation underwent a series of change, and it is summarized in Table 1.

As can be seen from Table 1, the trade restriction policy introduced in September 2015 was the most severe one, in which the transaction fee rose from $0.115 \%$ to $2.3 \%$ which greatly increased the cost of speculative transactions, which obviously has a tremendous impact on the market structure and market liquidity.

Figure 2 illustrates the trend of the closing price of CSI 300 index futures. It can be clearly seen that the market has experienced different market stages such as rising and plunging, spanning the entire turbulent period before and after the stock market crash, which indicated that the trading restriction policy has had a tremendous impact on the stock index future.

Figure 3 presents the trading volume of the CSI 300 index futures (logarithm). Compared with the trading volume before trade restriction (blue part), after the restriction policy was implemented on September 7 in 2015, due to the increase in margin, the cost of high-frequency transaction increased significantly, and the trading volume of the CSI 300 stock index futures (red part) was significantly reduced, indicating that the trading restriction policy has had a tremendous impact on stock index futures.

In order to effectively compare the impact of HFT on the liquidity of China's stock index futures market, this paper adopts an event-driven approach, taking the strictest trading restriction measures implemented in September in 2015 as the divided point. The experimental data samples are divided into two parts (see Table 2). The first part is from July 1, 2014, to September 2, 2015, and the second part ranges from September 7, 2015, to December 31, 2015. The first part can be simply thought as the active period of HFT, and the second part is nonactive because of the extreme expensive rate of the transaction fee, which is 20 times higher than before, and limited opening hands. Therefore, the first part of this period can be used to analyze how HFT affects the 


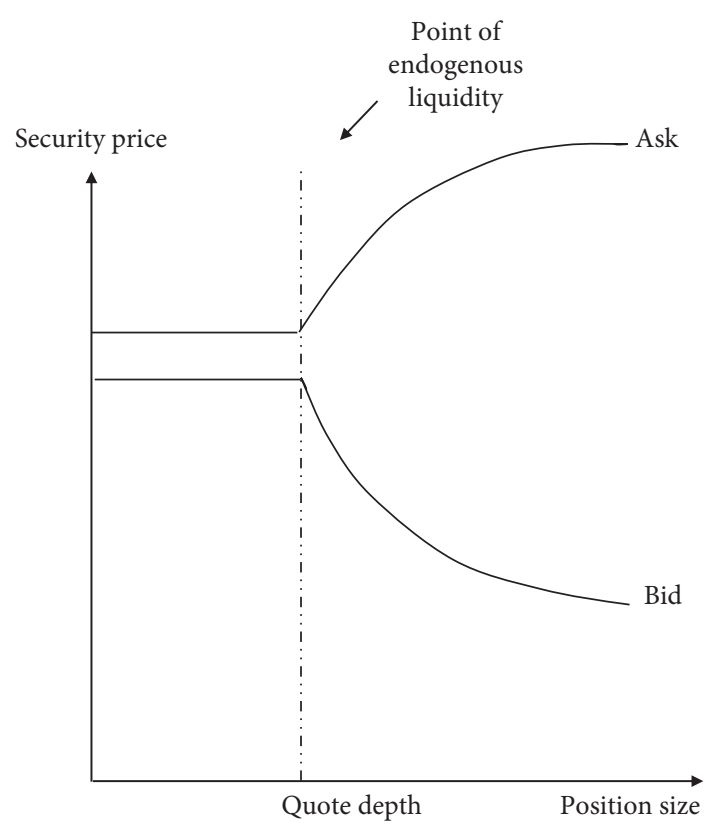

Figure 1: Relationship between position size and security size.

liquidity risk in the stock index future market, while the second part is used for comparison.

\section{Correlation of HFT and Market Liquidity}

In this section, the basic explanation of the VAR model will be given, followed by experiments on how HFT is correlated with market liquidity. The purpose of this section is to explore the relationship between HFT behaviour and market liquidity in the China stock index futures market using the trading agent index and liquidity indicators.

3.1. Correlation of HFT with Market Liquidity Based on VAR Model. Vector autoregression model (VAR) proposed by Sims in 1980 that has been widely used in the international finance and capital market is used in our study. The VAR model decomposes the variable $y$ into linear combinations of other variables and performs similar equation relations for other variables. It uses multiple simultaneous equations for modeling the relationship between variables. In each equation, endogenous variables will carry out regression of the lag value of all endogenous variables contained in the model, so as to estimate the dynamic relationship between all endogenous variables and make predictions. The specific formula is as follows:

$$
\begin{aligned}
y_{1 t}= & c_{1}+A_{1} y_{1 . t-1}+\cdots+A_{k} y_{1 . t-k}++B_{1} y_{2 . t-1}+\cdots \\
& +B_{k} y_{2 . t-k}+u_{1 t},
\end{aligned}
$$

where $y_{1 t}$ and $y_{2 t}$ are the $k$ vectors of endogenous variables. Based on the VAR model shown in equation (1), the correlation between $y_{1 t}$ and $y_{2 t}$ is established. $u_{t}$ is the random disturbance term of each equation, $c$ is the constant constraint term, and $A$ and $B$ are the coefficients of the lagging variable to be estimated.

Based on the VAR model introduced above, the correlation analysis between HFT and liquidity is given as follows:

$$
\begin{aligned}
\operatorname{algo}_{t}= & c_{1}+\sum_{j=1}^{3} \delta^{j} \operatorname{algo}_{t-j}+\alpha_{1} \operatorname{sprd}_{t}+\sum_{n=1}^{3} \Pi_{n} \operatorname{sprd}_{t-n}+v_{1, t} \operatorname{Size}_{t} \\
& +\psi_{1, t} \mathrm{Vol}_{t}+u_{1 t}, \\
\operatorname{sprd}_{t}= & c_{2}+\sum_{k=1}^{3} \Pi_{k} \mathrm{sprd}_{t-k}+\beta_{1} \operatorname{algo}_{t}+\sum_{m=1}^{3} \theta_{m} \mathrm{algo}_{t-m}+v_{2, t} \operatorname{Size}_{t} \\
& +\psi_{2, t} \mathrm{Vol}_{t}+u_{2 t},
\end{aligned}
$$

where algo $_{t}$ represents the trading amount of AT, which indicates the activeness of HFT behaviour; $\operatorname{sprd}_{t}$ as bid-ask spread is a collection of the liquidity measurement index including itself and the other two indicators as follows: $e S p r t_{t}$ represents the effective spread and $r S p r d{ }_{t}$ represents the realization spread, which indicates the income and net compensation of the liquidity provider like HF traders. Besides, $\mathrm{advSel}_{t}$ represents the adverse selection cost. There are also two control variables, Size $e_{t}$ is the transaction size and $\mathrm{Vol}_{t}$ is the volatility, which is determined by calculating historical volatility at the midpoint of the buy-sell price. Other variables including $\delta, \alpha, \beta, \Pi, \theta, \psi$, and $v$ are coefficients to be estimated.

For determining lag length $k$, we use two popular methods, the Akaike Information Criteria (AIC) [17] and the Schwartz Information Criteria (SIC) [18], to determine an appropriate lag period. Both AIC and SIC suggest a lag period $(k=3)$; therefore, the lag period $(k=3)$ is used in this study.

3.2. Correlation Analysis Result. Based on the VAR model introduced above, in this section, we compare the correlation analysis result to validate the correlation between HFT and market liquidity.

The experimental results are listed in Tables 3 and 4 , where the correlation between the HFT agent index Algo $_{t}$ and liquidity indicators including $\operatorname{sprd}_{t}, r \operatorname{Sprd}_{t}, e \operatorname{Sprd}_{t}$, and $\mathrm{AdvSel}_{t}$ is investigated, as shown in Model 1 to Model 4, respectively.

First, since the HFT activeness period is not known, we use HFT data during a long period spanning the HFT restriction policy (2014.07.01-2016.05.31) for correlation analysis, and the result is shown in Table 3.

In Table 3, the coefficient factor in Models 1-4 shows the correlation result between the HFT agent index algo $_{t}$ and different liquidity indicators including $\operatorname{sprd}_{t}, r \operatorname{Sprd}_{t}, e S \operatorname{Spd}_{t}$, and AdvSel ${ }_{t}$, respectively. Panel C (Models 1-4) shows the weak correlation between HFT and liquidity indicators, for which the coefficient of the lag term at time $t-1$ is -0.0103 , $-0.004,0.0333$, and 0.0069 at a confidence level 0.05 , respectively. It indicates that to some extent, HFT may correlate with the liquidity level from a large period which spans the trade restriction policy.

Considering a series of strict control measures on stock index futures trading since September 2015, especially the standard for setting the rule that abnormal trading is regarded as 10 hands per day, the activity of high-frequency trading has been greatly inhibited. 
TABLE 1: Trading rules of CSI 300 index futures (until the end of 2018).

\begin{tabular}{lccc}
\hline Time & Nonscheduled policy opening hands limit & Nonhedging (\%), hedging position trading margin (\%) & Transaction fee (\%) \\
\hline 2012.6 .29 & - & 12,12 & 0.035 \\
2014.9 .1 & - & 10,10 & 0.025 \\
2015.8 .28 & 600 & 20,10 & 0.115 \\
2015.8 .31 & 100 & 30,10 & 0.115 \\
2015.9 .7 & 10 & 40,20 & 23 \\
2017.2 .17 & 20 & 20,20 & 9.2 \\
2017.9 .18 & 20 & 15,15 & 6.9 \\
2018.12 .3 & 50 & 10,10 & 4.6 \\
\hline
\end{tabular}

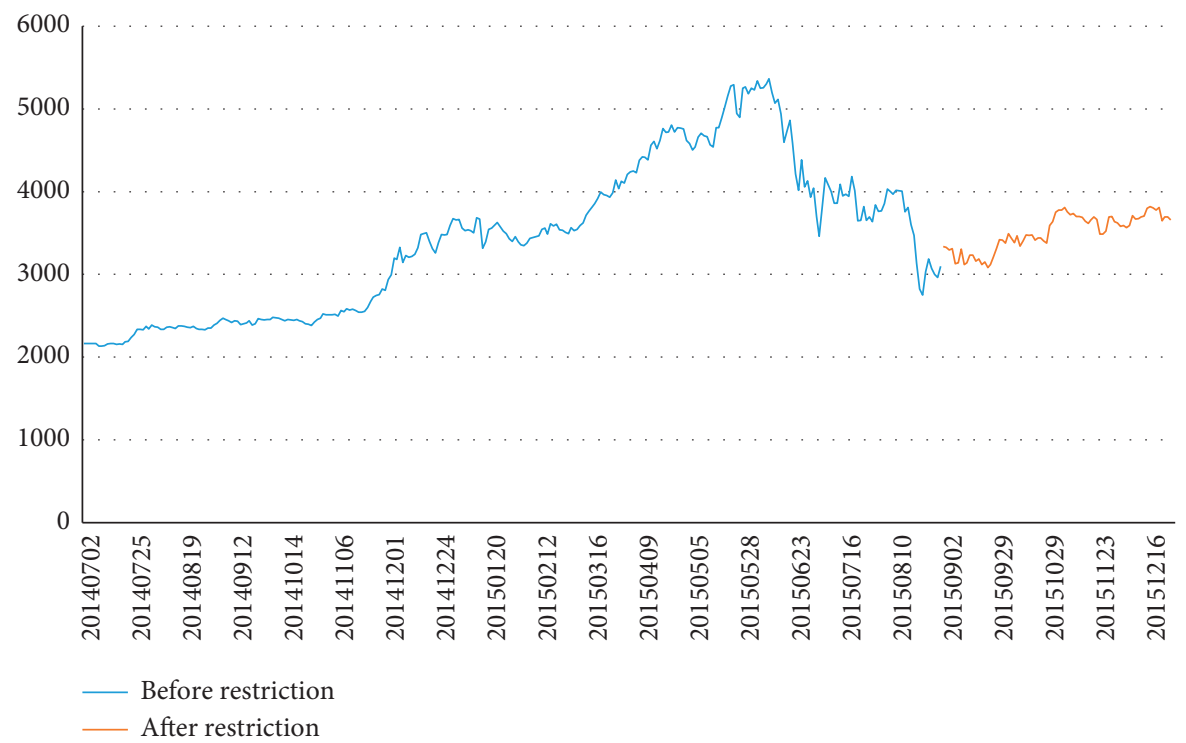

FIGURE 2: Closing price of CSI 300 index futures before and after trade restriction (2014.7.1-2015.12.31).

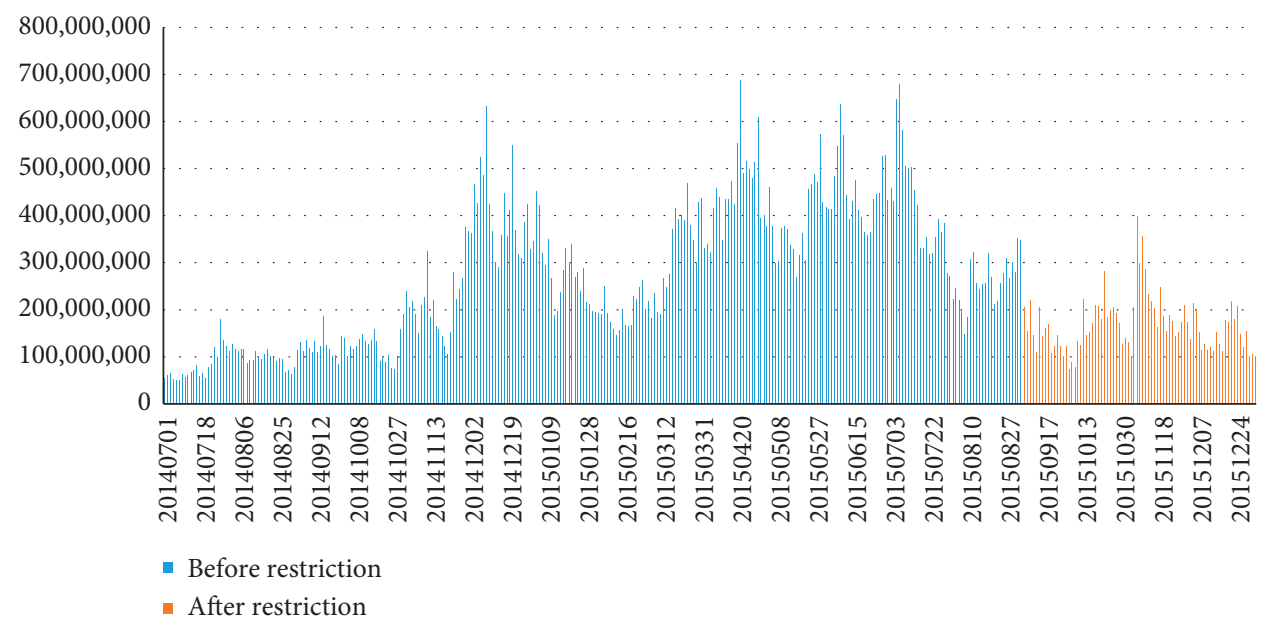

FIgURE 3: Volume of CSI 300 index futures before and after trade restriction (2014.7.1-2015.12.31).

TABle 2: Experimental data.

\begin{tabular}{lc}
\hline Before restriction & After restriction \\
\hline $2014.07 .1 \sim 2015.09 .02$ & $2015.09 .07 \sim 2015.12 .31$ \\
\hline
\end{tabular}

In this part, a more specific period range from July 1, 2014 , to September 2, 2015, is selected to further analyze the correlation between HFT and market liquidity. 
TABLE 3: Relationship between algorithmic trading and liquidity indicators (July 1, 2014-May 31, 2016).

\begin{tabular}{|c|c|c|c|c|c|c|c|}
\hline \multicolumn{4}{|c|}{ Model 1} & \multicolumn{4}{|c|}{ Model 2} \\
\hline \multicolumn{2}{|c|}{$\mathrm{Algo}_{t}$} & \multicolumn{2}{|c|}{$\operatorname{Sprd}_{t}$} & \multicolumn{2}{|c|}{$\mathrm{Algo}_{t}$} & \multicolumn{2}{|l|}{$r S p r d t$} \\
\hline$c_{1}$ & $0.2154^{* * *}$ & $c_{2}$ & $1.0475^{* * *}$ & $c_{1}$ & $0.1487^{* * *}$ & $c_{2}$ & $2.8018^{* * *}$ \\
\hline \multicolumn{8}{|c|}{ Panel A: contemporaneous variables } \\
\hline $\operatorname{Sprd}_{t}$ & $0.0236^{* * *}$ & $\mathrm{Algo}_{t}$ & $0.0825^{* * *}$ & $r \operatorname{Sprd}_{t}$ & $0.005^{* * *}$ & $\mathrm{Algo}_{t}$ & $1.3549^{* * *}$ \\
\hline \multicolumn{8}{|c|}{ Panel B: autoregressive lagged variables } \\
\hline $\mathrm{Algo}_{t-1}$ & $0.5215^{* * *}$ & $\operatorname{Sprd}_{t-1}$ & $0.6896^{* * *}$ & $\mathrm{Algo}_{t-1}$ & 0.5214 & $r S p r d d_{t-1}$ & $0.2288^{* * *}$ \\
\hline $\mathrm{Algo}_{t-2}$ & $0.1724^{* * *}$ & Sprd $_{t-2}$ & $0.1126^{* * *}$ & $\mathrm{Algo}_{t-2}$ & $0.1878^{*}$ & $r \mathrm{Sprd}_{t-2}$ & $0.0439^{* * *}$ \\
\hline $\mathrm{Algo}_{t-3}$ & $0.1648^{* * *}$ & Sprd $_{t-3}$ & $0.1029^{* * *}$ & $\mathrm{Algo}_{t-3}$ & 0.1598 & $r \operatorname{Sprd}_{t-3}$ & $0.1057^{* * *}$ \\
\hline \multicolumn{8}{|c|}{ Panel C: cross effect of lagged variables } \\
\hline $\operatorname{Sprd}_{t-1}$ & -0.0103 & $\mathrm{Algo}_{t-1}$ & $-0.0747^{* * *}$ & $r \operatorname{Sprd}_{t-1}$ & -0.0004 & $\mathrm{Algo}_{t-1}$ & -0.2771 \\
\hline Sprd $_{t-2}$ & -0.0043 & $\mathrm{Algo}_{t-2}$ & 0.0319 & $r \operatorname{Sprd}_{t-2}$ & $0.0041^{* * *}$ & $\mathrm{Algo}_{t-2}$ & $-0.4124^{*}$ \\
\hline $\operatorname{Sprd}_{t-3}$ & -0.0015 & $\mathrm{Algo}_{t-3}$ & 0.0311 & $r \operatorname{Sprd}_{t-3}$ & $0.0022^{* * *}$ & $\mathrm{Algo}_{t-3}$ & -0.3044 \\
\hline \multicolumn{8}{|c|}{ Panel D: control variables } \\
\hline $\mathrm{Vol}_{t}$ & $-0.0251^{* * *}$ & $\mathrm{Vol}_{t}$ & $0.0232^{* * *}$ & $\mathrm{Vol}_{t}$ & $-0.0379^{* * *}$ & $\mathrm{Vol}_{t}$ & $1.1059^{* * *}$ \\
\hline $\mathrm{Size}_{t}$ & $-0.4785^{* * *}$ & Size $_{t}$ & $-0.4618^{* * *}$ & Size $_{t}$ & $-0.4203^{* * *}$ & Size $_{t}$ & $0.0059^{* * *}$ \\
\hline \multicolumn{4}{|c|}{ Model 3} & \multicolumn{4}{|c|}{ Model 4} \\
\hline \multicolumn{2}{|c|}{$\mathrm{Algo}_{t}$} & \multicolumn{2}{|c|}{$e \operatorname{Sprd}_{t}$} & \multicolumn{2}{|c|}{$\mathrm{Algo}_{t}$} & \multicolumn{2}{|c|}{$\mathrm{AdvSel}_{t}$} \\
\hline$c_{1}$ & $0.1171^{* *}$ & $c_{2}$ & $0.0692^{* * *}$ & $c_{1}$ & $0.1383^{* * *}$ & $c_{2}$ & $1.8431^{* * *}$ \\
\hline \multicolumn{8}{|c|}{ Panel A: contemporaneous variables } \\
\hline$e$ Sprd ${ }_{t}$ & $0.1636^{*}$ & $\mathrm{Algo}_{t}$ & $0.0032^{*}$ & $\mathrm{AdvSel}_{t}$ & $-0.0055^{* * *}$ & $\mathrm{Algo}_{t}$ & $-0.6859^{* * *}$ \\
\hline \multicolumn{8}{|c|}{ Panel B: autoregressive lagged variables } \\
\hline $\mathrm{Algo}_{t-1}$ & $0.5201^{* * *}$ & $e S p r d{ }_{t-1}$ & $0.6776^{* * *}$ & $\mathrm{Algo}_{t-1}$ & $0.5372^{* * *}$ & $\mathrm{AdvSel}_{t-1}$ & $0.2436^{* * *}$ \\
\hline $\mathrm{Algo}_{t-2}$ & $0.1723^{* * *}$ & $e \operatorname{Sprd}_{t-2}$ & $0.1199^{* * *}$ & $\mathrm{Algo}_{t-2}$ & $0.1869^{* * *}$ & $\mathrm{AdvSel}_{t-2}$ & $0.1019^{* * *}$ \\
\hline $\mathrm{Algo}_{t-3}$ & $0.1544^{* * *}$ & $e \operatorname{Sprd}_{t-3}$ & $0.1029^{* * *}$ & $\mathrm{Algo}_{t-3}$ & $0.1600^{* * *}$ & $\mathrm{AdvSel}_{t-3}$ & $0.0906^{* * *}$ \\
\hline \multicolumn{8}{|c|}{ Panel C: cross effect of lagged variables } \\
\hline$e \operatorname{Sprd}_{t-1}$ & 0.0333 & $\mathrm{Algo}_{t-1}$ & $-0.0038^{*}$ & $\mathrm{AdvSel}_{t-1}$ & $0.0069^{* * *}$ & $\mathrm{Algo}_{t-1}$ & $0.9865^{* * *}$ \\
\hline$e \operatorname{Sprd}_{t-2}$ & 0.0737 & $\mathrm{Algo}_{t-2}$ & $0.0036^{*}$ & $\mathrm{AdvSel}_{t-2}$ & $0.0049^{* * *}$ & $\mathrm{Algo}_{t-2}$ & -0.1064 \\
\hline$e S p r d d_{t-3}$ & -0.1213 & $\mathrm{Algo}_{t-3}$ & $0.0038^{* *}$ & $\mathrm{AdvSel}_{t-3}$ & $0.0024^{* * *}$ & $\mathrm{Algo}_{t-3}$ & $0.3277^{* *}$ \\
\hline \multicolumn{8}{|c|}{ Panel D: control variables } \\
\hline $\mathrm{Vol}_{t}$ & $-0.0257^{* * *}$ & $\mathrm{Vol}_{t}$ & $0.0017^{* * *}$ & $\mathrm{Vol}_{t}$ & $-0.0307^{* * *}$ & $\mathrm{Vol}_{t}$ & $1.1320^{* * *}$ \\
\hline Size $_{t}$ & $-0.4324^{* * *}$ & Size $_{t}$ & $-0.0237^{* * *}$ & Size $_{t}$ & $-0.3814^{* * *}$ & Size $_{t}$ & $0.7659^{* * *}$ \\
\hline
\end{tabular}

Compared with Table 3, in Table 4, the coefficients in Panel C from lag term 1 to 3 all shows that the correlation between HFT and liquidity indicators increases to $0.1643,0.19926,0.0094$, and 0.0094 at a confidence level 0.05 , which is far greater than the corresponding coefficients in Table 3 . It means before the restriction policy, HFT is obviously more active, and the correlation between market liquidity level is stronger. In Panel D, all the liquidity indicators show a positive correlation with volatility, indicating that when the volatility is higher, the liquidity is higher. AT activity is negatively correlated with volatility and trading size, indicating that algorithmic traders tend to trade when volatility and trading size are smaller. This means that algorithmic traders like HF traders tend to enter the market strategically with lower transaction costs and less information asymmetry, which indicates that HFT improves the market liquidity level.

Based on the above experiments, it can be concluded that before trade restriction when HFT behaviour is active, more algorithm-based trades are correlated with market liquidity indicated by a larger absolute spread $\left(\operatorname{Sprd}_{t}\right)$, larger effective spread $\left(e \operatorname{Sprd}_{t}\right)$, larger realized spread $\left(r \operatorname{Sprd}_{t}\right)$, and less adverse selection $\left(\operatorname{advSel}_{t}\right)$. It also indicates that the trade restriction policy greatly changes the activeness of HFT behaviour.

\section{Impact of HFT on Liquidity Risk}

The correlation between HFT and liquidity is demonstrated by the VAR model in Section 3. To further analyze how HFT influences exogenous liquidity risk before and after trade restriction, the LVaR is introduced in this section.

4.1. Liquidity Risk Value Measurement Model. The classis $\mathrm{VaR}$ model and the improved LVaR model-added market factors are introduced in this section, which is the basis for analyzing the impact of HFT on liquidity risk with the LVaR model in the next section.

4.1.1. VaR Model. In China's order-driven futures market, investors' trading orders are directly paired through the trading 
TABLE 4: Relationship between algorithmic trading and liquidity indicators (September 7, 2015-December 31, 2015, excluding opening and closing time windows).

\begin{tabular}{|c|c|c|c|c|c|c|c|}
\hline \multicolumn{4}{|c|}{ Model 1} & \multicolumn{4}{|c|}{ Model 2} \\
\hline \multicolumn{2}{|c|}{$\mathrm{Algo}_{t}$} & \multicolumn{2}{|c|}{$\operatorname{Sprd}_{t}$} & \multicolumn{2}{|c|}{$\mathrm{Algo}_{t}$} & \multicolumn{2}{|c|}{$r \operatorname{Sprd}_{t}$} \\
\hline$c_{1}$ & 0.0651 & $c_{2}$ & $0.6159^{* * *}$ & $c_{1}$ & $0.3095^{* * *}$ & $c_{2}$ & -0.7202 \\
\hline \multicolumn{8}{|c|}{ Panel A: contemporaneous variables } \\
\hline Sprd $_{t}$ & $-0.1405^{* * *}$ & $\mathrm{Algo}_{t}$ & $-0.0928^{* * *}$ & $r \operatorname{Sprd}_{t}$ & $-0.0157^{* * *}$ & $\mathrm{Algo}_{t}$ & $-2.2388^{* * *}$ \\
\hline \multicolumn{8}{|c|}{ Panel B: autoregressive lagged variables } \\
\hline $\mathrm{Algo}_{t-1}$ & $0.5563^{* * *}$ & Sprd $_{t-1}$ & $0.6350^{* * *}$ & $\mathrm{Algo}_{t-1}$ & $0.5653^{* * *}$ & $r \mathrm{Sprd}_{t-1}$ & $0.2318^{* * *}$ \\
\hline $\mathrm{Algo}_{t-2}$ & $0.1956^{* * *}$ & $\operatorname{Sprd}_{t-2}$ & $-0.0413^{*}$ & $\mathrm{Algo}_{t-2}$ & $0.2185^{* * *}$ & $r \operatorname{Sprd}_{t-2}$ & $0.1695^{* * *}$ \\
\hline $\mathrm{Algo}_{t-3}$ & $0.1648^{* * *}$ & $\operatorname{Sprd}_{t-3}$ & $0.1945^{* * *}$ & $\mathrm{Algo}_{t-3}$ & $0.1528^{* * *}$ & $r S p r d d_{t-3}$ & $0.0814^{* * *}$ \\
\hline \multicolumn{8}{|c|}{ Panel C: cross effect of lagged variables } \\
\hline Sprd $_{t-1}$ & $0.1643^{* * *}$ & $\operatorname{Algo}_{t-1}$ & $0.0332^{* *}$ & $r S p r d d_{t-1}$ & $0.0094^{* * *}$ & $\mathrm{Algo}_{t-1}$ & $1.5264^{* * *}$ \\
\hline $\operatorname{Sprd}_{t-2}$ & $0.0582^{* *}$ & $\operatorname{Algo}_{t-2}$ & 0.0154 & $r \operatorname{Sprd}_{t-2}$ & $0.0098^{* * *}$ & $\mathrm{Algo}_{t-2}$ & $0.6386^{* *}$ \\
\hline $\operatorname{Sprd}_{t-3}$ & 0.0257 & $\mathrm{Algo}_{t-3}$ & $0.0404^{* * *}$ & $r S p r d d_{t-3}$ & $0.0028^{* *}$ & $\mathrm{Algo}_{t-3}$ & $0.3712^{*}$ \\
\hline \multicolumn{8}{|c|}{ Panel D: control variables } \\
\hline $\mathrm{Vol}_{t}$ & $-0.0312^{* * *}$ & $\mathrm{Vol}_{t}$ & $0.0291^{* * *}$ & $\mathrm{Vol}_{t}$ & $-0.0220^{* * *}$ & $\mathrm{Vol}_{t}$ & $1.0666^{* * *}$ \\
\hline Size $_{t}$ & $-0.2917^{* * *}$ & Size $_{t}$ & $-0.2738^{* * *}$ & Size $_{t}$ & $-0.3589^{* * *}$ & Size $_{t}$ & $1.8179^{* * *}$ \\
\hline \multicolumn{4}{|c|}{ Model 3} & \multicolumn{4}{|c|}{ Model 4} \\
\hline \multicolumn{2}{|c|}{$\mathrm{Algo}_{t}$} & \multicolumn{2}{|c|}{$e$ Sprd ${ }_{t}$} & \multicolumn{2}{|c|}{$\mathrm{Algo}_{t}$} & \multicolumn{2}{|c|}{$\mathrm{AdvSel}_{t}$} \\
\hline$c_{1}$ & 0.1261 & $c_{2}$ & $0.0242^{* * *}$ & $c_{1}$ & $0.3069^{* * *}$ & $c_{2}$ & -0.7350 \\
\hline \multicolumn{8}{|c|}{ Panel A: contemporaneous variables } \\
\hline$e \operatorname{Sprd}_{t}$ & $-1.5548^{* * *}$ & $\mathrm{Algo}_{t}$ & $-0.0069^{* * *}$ & $\mathrm{AdvSel}_{t}$ & $-0.0157^{* * *}$ & $\mathrm{Algo}_{t}$ & $-2.2388^{* * *}$ \\
\hline \multicolumn{8}{|c|}{ Panel B: autoregressive lagged variables } \\
\hline $\mathrm{Algo}_{t-1}$ & $0.5478^{* * *}$ & $e \operatorname{Sprd}_{t-1}$ & $0.6694^{* * *}$ & $\mathrm{Algo}_{t-1}$ & $0.5653^{* * *}$ & $\mathrm{AdvSel}_{t-1}$ & $0.2319^{* * *}$ \\
\hline $\mathrm{Algo}_{t-2}$ & $0.1894^{* * *}$ & $e \operatorname{Sprd}_{t-2}$ & $-0.0705^{* * *}$ & $\mathrm{Algo}_{t-2}$ & $0.2183^{* * *}$ & AdvSel $_{t-2}$ & $0.1694^{* * *}$ \\
\hline $\mathrm{Algo}_{t-3}$ & $0.1598^{* * *}$ & $e \operatorname{Sprd}_{t-3}$ & $0.2118^{* * *}$ & $\mathrm{Algo}_{t-3}$ & $0.1529^{* * *}$ & $\operatorname{AdvSel}_{t-3}$ & $0.0816^{* * *}$ \\
\hline \multicolumn{8}{|c|}{ Panel C: cross effect of lagged variables } \\
\hline$e \operatorname{sprd}_{t-1}$ & $1.9926^{* * *}$ & $\mathrm{Algo}_{t-1}$ & $0.0047^{* * *}$ & $\operatorname{AdvSel}_{t-1}$ & $0.0094^{* * *}$ & $\mathrm{Algo}_{t-1}$ & $1.5239^{* * *}$ \\
\hline$e \operatorname{Sprd}_{t-2}$ & $0.7224^{* *}$ & $\mathrm{Algo}_{t-2}$ & 0.0015 & $\operatorname{AdvSel}_{t-2}$ & $0.0098^{* * *}$ & $\mathrm{Algo}_{t-2}$ & $0.6369^{* *}$ \\
\hline$e \operatorname{Sprd}_{t-3}$ & $0.4966^{*}$ & $\mathrm{Algo}_{t-3}$ & $0.0033^{* *}$ & $\mathrm{AdvSel}_{t-3}$ & $0.0028^{* *}$ & $\mathrm{Algo}_{t-3}$ & $0.3732^{*}$ \\
\hline \multicolumn{8}{|c|}{ Panel D: control variables } \\
\hline $\mathrm{Vol}_{t}$ & $-0.0307^{* * *}$ & $\mathrm{Vol}_{t}$ & $0.0018^{* * *}$ & $\mathrm{Vol}_{t}$ & $-0.0220^{* * *}$ & $\mathrm{Vol}_{t}$ & $1.0658^{* * *}$ \\
\hline Size $_{t}$ & $-0.4101^{* * *}$ & Size $_{t}$ & $-0.0069^{* * *}$ & Size $_{t}$ & $-0.3589^{* * *}$ & Size $_{t}$ & $1.8174^{* * *}$ \\
\hline
\end{tabular}

system, and the flow of trading commissions is the fundamental driving force of liquidity.

Supposing that the investor's logarithmic return at time $t$ in the long position dominant contract of the stock index futures conforms to the random walk process, then

$$
P_{t}=P_{t-1} e^{\mu+\sigma \varepsilon_{t}},
$$

in which $P_{t}$ is the midpoint price of the quote at time $t, \mu$ is the drift rate, $\sigma$ is the standard deviation of the return rate, $\varepsilon_{t}$ is the random disturbance term, and there is $\varepsilon_{t} \sim N(0,1)$. Assuming an offset rate of 0 , the 1-day short position $\mathrm{VaR}$ at 99\% confidence level under a standard normal distribution can be simplified as

$$
\operatorname{VaR}=P_{t}\left(1-e^{-2.33 \theta \sigma_{t}}\right)
$$

There will be a large price change when the dominant contract rolls, so $\mathrm{VaR}$ in the form of yield is more reasonable as the following:

$$
\operatorname{VaR}=1-e^{-2.33 \theta \sigma_{t}},
$$

where $\theta$ is the correction factor, and the calculation formula is

$$
\theta=1+\phi \ln \left(\frac{k}{3}\right)
$$

where $k$ is the kurtosis value of the return rate and $\varnothing$ is a constant which can be obtained by regression of equation (5). When the return is a normal distribution, $k=3$, so $\theta=1$. The correction factor has a value greater than 1 when the return is a peak thick tail shape, which is used to correct the undervalued value risk.

4.1.2. LVaR Model. The traditional VaR model has an implicit assumption that regardless of the trading position of investors, the transaction can be completed at a fixed market 
price within a fixed period of time [19]. Obviously, the VaR model ignores the fluctuations in market prices and spreads that large deals can bring. In order to solve problems mentioned above, a liquidity risk estimation model is proposed.

Hisata and Yamai [12] believe that the uncertainty of bid-ask spread can be used as a performance of liquidity risk. Therefore, they introduced the volatility indicators of bidask spread to exogenous cost of liquidity (ECL) and proposed the liquidity-adjusted value at risk (LVaR) model.

The exogenous liquidity cost defined by the model is

$$
\mathrm{ECL}=P_{t} \frac{\left(\bar{S}+a \tilde{\sigma_{t}}\right)}{2},
$$

where $P_{t}$ is the midpoint price of the quoted price at time $t, \bar{S}$ is the mean of the relative bid spread, $\widetilde{\sigma}_{t}$ is the volatility of the relative bid spread, and $\alpha$ is the scale factor. Therefore, the ECL is the maximum loss that can be caused by the exogenous fluidity risk when there is a spread at $\alpha$ confidence level. Correspondingly, the maximum loss that may be caused by the exogenous liquidity risk of multiple short commodities expressed by the yield is

$$
\mathrm{ECL}=\frac{\left(\bar{s}+a \tilde{\sigma}_{t}\right)}{2} .
$$

The LVaR model is a liquidity-adjusted VaR model that adds the exogenous liquidity cost to VaR. Adding exogenous liquidity risk to the traditional VaR model is to correct the traditional liquidity risk prediction model:

$$
\mathrm{LVaR}=P_{t}\left(1-e^{-2.33 \theta \sigma_{t}}\right)+P_{t} \frac{\left(\bar{S}+a \tilde{\sigma_{t}}\right)}{2} .
$$

The liquidity risk ratio can be obtained by removing the absolute price $P_{t}$ :

$$
\mathrm{LVaR}=\left(1-e^{-2.33 \theta \sigma_{t}}\right)+\frac{\left(\bar{S}+a \tilde{\sigma_{t}}\right)}{2} .
$$

As can be seen from equation (11), the following parameters are necessary to calculate the LVaR model:

(1) $\bar{S}$ is the mean of the relative bid spread

(2) $\tilde{\sigma}_{t}$ is the volatility of the relative bid spread

(3) $\alpha$ is the scale factor

(4) $\widetilde{\sigma}$ is the volatility of the return rate

\subsection{Impact Measurement with LVaR Model}

4.2.1. Estimation of Volatility in Yield. The stock price index is usually a nonstationary time series, while the yield series shows stationarity. Therefore, this article takes the logarithmic yield of the CSI 300 index futures as the research object with following formula to calculate the yield:

$$
\mathrm{R}_{\mathrm{t}}=\ln \left(P_{t}\right)-\ln \left(P_{t-1}\right),
$$

where $R_{t}$ represents the logarithmic yield for period $t$ and $P_{t}$ and $P_{t-1}$ represent the closing price of the $t_{t h}$ and $(t-1)_{\text {th }}$ periods of the CSI 300 index futures.

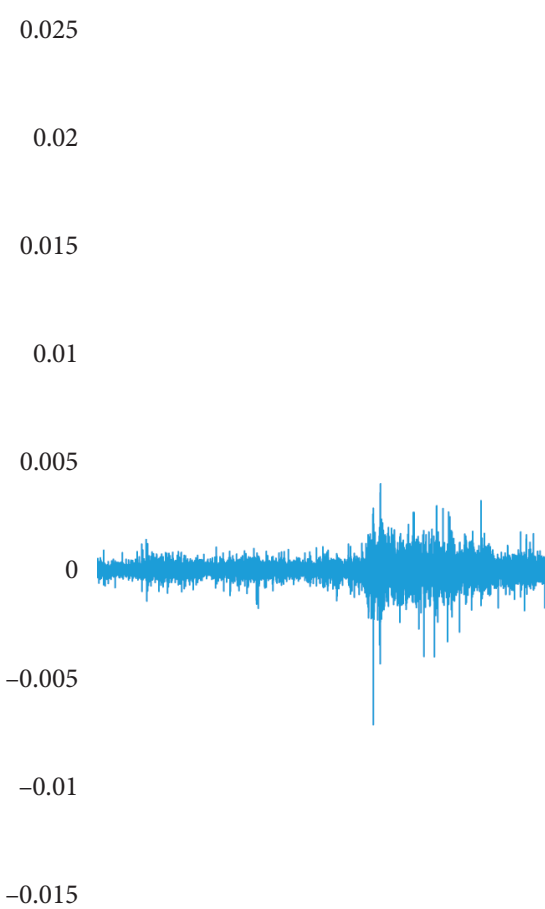

FIgURE 4: Return yield curve at minute level of CSI 300 index futures.

It is necessary to perform a stationary examination on the yield series before constructing the yield and volatility prediction model. Figure 4 shows the yield curve of the CSI 300 index futures at minute-level from July 1, 2014, to December 31, 2015. It can be observed that the yield curve fluctuates roughly within a range, indicating that the yield series may be stationary.

In order to further analyze the stationarity of the yield, the ADF unit root test is used. To be specific, if the ADF statistic value is less than the critical value at the given significance level, the null hypothesis that at least one unit root exists is rejected, indicating the time series is stationary, and conversely the time series is nonstationary. According to the unit root test result shown as -7.52 , it can be concluded that the yield series of the CSI 300 index futures is stationary at the $5 \%$ significance level and can be fitted by regression.

It is necessary to first perform the ARCH effect test on the residual of the mean value equation of the yield series before modeling the volatility of the CSI 300 index futures return yield. Only the yield series satisfying the ARCH effect can use the GARCH model to analyze the volatility rate. In this paper, the Ljung-Box examination [20] is used to examine the autocorrelation of the square series of the yield. The statistic $p$ value of the test is 0 , so the null hypothesis that the square series of yield is white noise (no autocorrelation) can be rejected, indicating that the original series (the yield) does have an ARCH effect.

After proving that the yield series satisfies the stationary and ARCH effect, volatility is analyzed with the GARCH model in this section. Considering that the order of the GARCH model is hard to determine, the low-order GARCH $(1,1)$ model is selected.

First, the AR(3) model is used to fit the yield series of the CSI 300 index futures active period. Then, the volatility rate 
TABLE 5: The fitting results of the GARCH $(1,1)$ model in the active stage of HFT.

\begin{tabular}{lcccc}
\hline \multicolumn{5}{l}{ Mean equation $\mathrm{AR}(3)$} \\
\hline Variable & Coefficient & Std. error & $t$-statistic & Prob \\
Const & $-3.4198 \mathrm{e}-07$ & $1.094 \mathrm{e}-05$ & $-3.127 \mathrm{e}-02$ & 0.975 \\
AR(1) & 0.0262 & $4.203 \mathrm{e}-03$ & 6.228 & $1.794 \mathrm{e}-02$ \\
$\mathrm{AR}(2)$ & $1.3168 \mathrm{e}-03$ & $4.479 \mathrm{e}-03$ & 0.294 & $-7.461 \mathrm{e}-03$ \\
$\mathrm{AR}(3)$ & $-1.4527 \mathrm{e}-03$ & $4.581 \mathrm{e}-03$ & -0.317 & $-1.043 \mathrm{e}-02$ \\
\hline Volatility & equation GARCH(1,1) & & \\
Omega & $4.4823 \mathrm{e}-08$ & $3.940 \mathrm{e}-12$ & 3.956 & $4.482 \mathrm{e}-08$ \\
Alpha(1) & 0.0500 & $9.623 \mathrm{e}-03$ & 5.196 & $3.114 \mathrm{e}-02$ \\
Beta(1) & 0.7800 & $4.204 \mathrm{e}-02$ & 18.554 & 0.698 \\
\hline
\end{tabular}

of residual is modeled with the GARCH model. The results are shown in Table 5.

According to the fitting results in Table 5, the mean equation can be derived as

$$
R_{t}=-3.7198 e^{-7}+0.0262 R_{t-1}+0.0013168 R_{t-2}-0.0014527 R_{t-3} .
$$

Volatility (conditional variance) equation:

$$
\sigma^{2}=4.4823 e^{-8}+0.05 \varepsilon_{t-1}^{2}+0.78 \sigma_{t-1}^{2} .
$$

It can be observed in Table 5 that the GARCH $(1,1)$ model is remarkable at the $5 \%$ significance level, indicating that the fitting effect is satisfactory.

As shown in Table 6, the mean and volatility equations during the period that HFT is restricted can be calculated with the same method.

According to the fitting results in Table 6, the mean equation of the yield during the period that HFT is restricted can be obtained as

$$
R_{t}=2.5018 e^{-5}+-0.0338 R_{t-1}+0.0328 R_{t-2}+0.0072765 R_{t-3} .
$$

Volatility rate (conditional variance) equation:

$$
\sigma^{2}=2.5232 e^{-8}+0.125 \varepsilon_{t-1}^{2}+0.93 \sigma_{t-1}^{2} .
$$

Similarly, the volatility rate equation of relative spread before and after trade restriction can be calculated separately as follows:

$$
\begin{aligned}
& \sigma^{2}=4.2112 e^{-11}+0.2 \varepsilon_{t-1}^{2}+0.78 \sigma_{t-1}^{2}, \\
& \sigma^{2}=4.3319 e^{-10}+0.1 \varepsilon_{t-1}^{2}+0.88 \sigma_{t-1}^{2} .
\end{aligned}
$$

4.2.2. Estimation of the Correction Factor $\theta$. To estimate the value of $\theta$, we first calculate the yield VaR of the CSI 300 index futures from the one year before trade restriction to one year after trade restriction at the $99 \%$ confidence with the historical simulation method and estimate $\sigma_{t}$ with the GARCH model. With the above two parameters, regression to equation (6) obtains that $\theta$ of the CSI 300 index futures is 1.046. At the same time, the kurtosis analysis of the yield inferred that the kurtosis value is 3.50. After that, the above
TABLE 6: The fitting results of the GARCH $(1,1)$ model during the HFT restricted period.

\begin{tabular}{lcccc}
\hline \multicolumn{5}{l}{ Mean equation AR(3) } \\
\hline Variable & Coefficient & Std. error & $t$-statistic & Prob \\
Const & $2.5018 \mathrm{e}-05$ & $7.060 \mathrm{e}-06$ & 3.5440 & $3.943 \mathrm{e}-04$ \\
AR(1) & -0.0338 & $6.850 \mathrm{e}-03$ & -4.940 & $-4.726 \mathrm{e}-02$ \\
AR(2) & 0.0328 & $6.966 \mathrm{e}-03$ & 4.712 & $1.917 \mathrm{e}-02$ \\
AR(3) & $7.2765 \mathrm{e}-03$ & $7.090 \mathrm{e}-03$ & 1.026 & $-6.620 \mathrm{e}-03$ \\
\hline Volatility & equation GARCH(1,1) & & \\
Omega & $2.5232 \mathrm{e}-08$ & $3.641 \mathrm{e}-12$ & 6930.443 & $2.522 \mathrm{e}-08$ \\
Alpha(1) & 0.0125 & $5.688 \mathrm{e}-03$ & 2.197 & $1.351 \mathrm{e}-03$ \\
Beta(1) & 0.9300 & $3.293 \mathrm{e}-03$ & 282.391 & 0.924 \\
\hline
\end{tabular}

two values are brought into equation (7), which inferred that $\phi$ at the $99 \%$ confidence level is 0.3 .

4.2.3. Exogenous Liquidity Cost. The scale factor $\alpha$ in the exogenous liquidity cost model is an essential element of the calculation. In this section, the CSI 300 index futures $\alpha$ is obtained at 5.134 after regression to 20 with ECL, $\bar{S}$, and $\widetilde{\sigma}_{t}$ calculated by HFT data from July 1, 2014, to December 31, 2015.

Therefore, the calculation formula for the exogenous liquidity cost before trade restriction is

$$
\mathrm{ECL}=\frac{1}{2}\left(1.12 e^{-4}+5.134 \widetilde{\sigma}_{t}\right) .
$$

The calculation formula for the exogenous liquidity cost after trade restriction is

$$
\mathrm{ECL}=\frac{1}{2}\left(3.86 e^{-4}+5.134 \widetilde{\sigma}_{t}\right) .
$$

The LVaR model is a liquidity-adjusted VaR model that adds the exogenous liquidity cost to VaR. Adding exogenous liquidity risk to the traditional $\mathrm{VaR}$ model is to correct the traditional low liquidity risk prediction model:

$$
L V a R=P_{t}\left(1-e^{-2.33 \theta \sigma_{t}}\right)+P_{t} \frac{\left(\bar{s}+a \sigma_{t}\right)}{2} .
$$

The liquidity risk ratio can be obtained by removing the absolute price $P_{t}$ :

$$
L V a R=\left(1-e^{-2.33 \theta \sigma_{t}}\right)+\frac{\left(\bar{s}+a \sigma_{t}\right)}{2} .
$$

4.3. Comparison of Impact of HFTon Liquidity Risk before and after Stock Index Futures Trade Restriction. Firstly, $\widetilde{\sigma}$ and $\widetilde{\sigma}_{t}$ obtained by the yield equation and the relative price volatility rate equation constructed based on the GARCH model in the previous section and the correction factor $\theta$ can be used to calculate the corresponding VaR value. Then, the value of exogenous liquidity cost ECL is calculated with the scale factor $\alpha$ and the relative price volatility rate equation. Finally, the final LVaR value is obtained according to (22).

$\mathrm{ECL} / \mathrm{LVaR}$ is defined as the exogenous liquidity risk ratio, an indicator that shows the proportion of liquidity risk in all risks of CSI 300 index futures. It can be seen from 


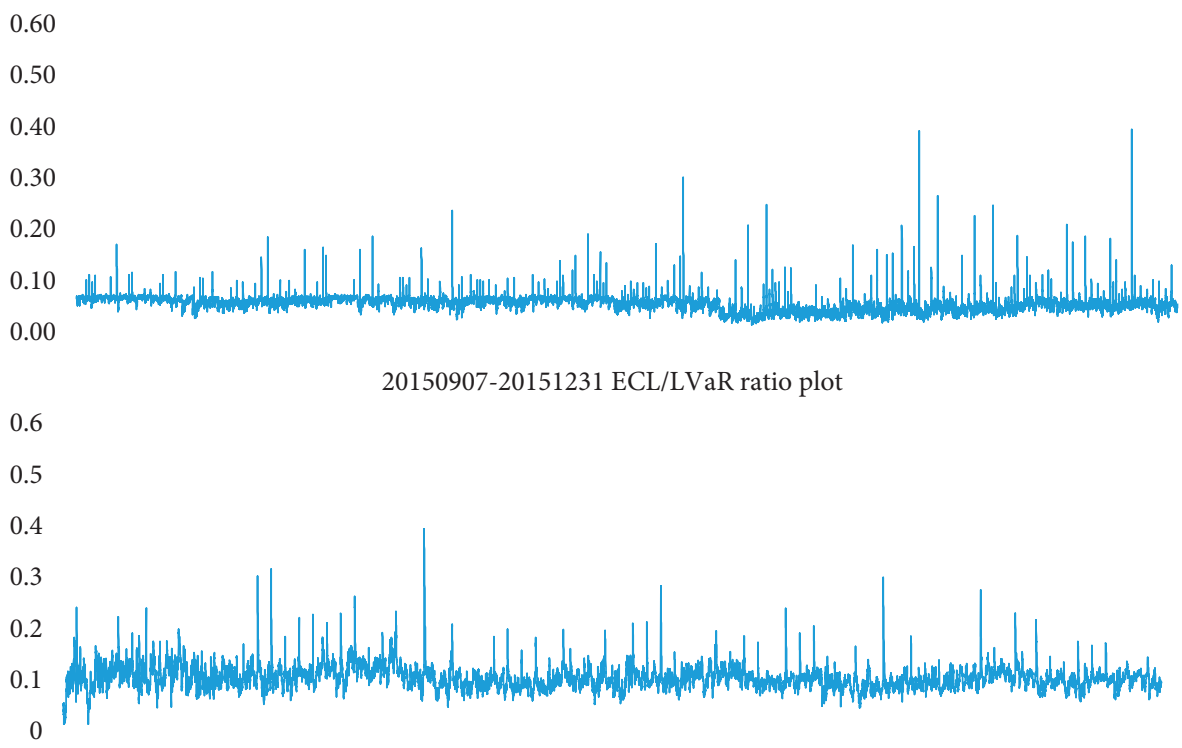

FIGURE 5: ECL/LVaR ratio chart before trade restriction of CSI 300 index futures (above) and after trade restriction (below) (minute level).
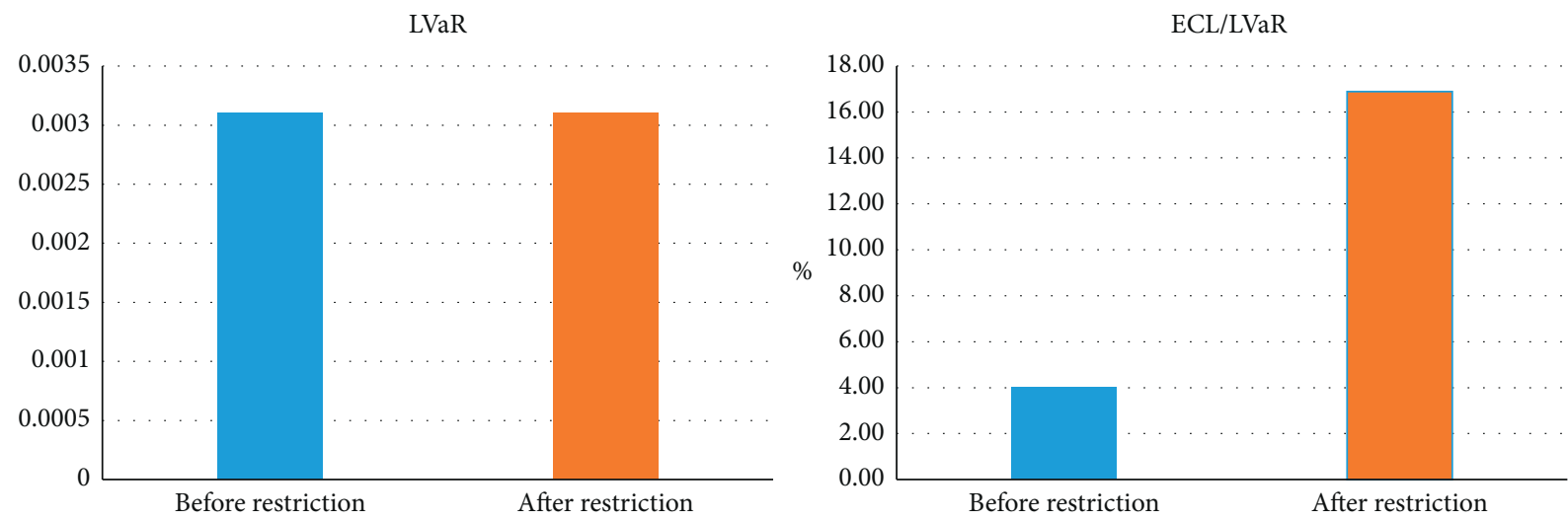

Figure 6: Comparison of ECL/LVaR and LVaR influenced by HFT behaviour.

Figure 5 that the mean value and volatility of ECL/LVaR rose obviously after the trade restriction policy.

To further analyze the impact on liquidity risk by HFT, Figure 6 shows the average value of ECL/LVaR and LVaR before and after trade restriction. It is interesting to find that the HFT restriction policy did not lift the liquidity risk level (LVaR), both remain at 0.0031 . However, the proportion of exogenous liquidity risk in LVaR rose from $4.04 \%$ before trade restriction to $16.89 \%$ after trade restriction. It means that the trade restriction policy significantly increases the exogenous liquidity risk. If the period before trade restriction is regarded as the environment in which HFT behaviour may occur, the period after trade restriction is regarded as the environment in which no HFT behaviour occurs. The findings indicate that the behaviour of HFT can effectively reduce the proportion of exogenous liquidity risk in the overall risk in the Chinese stock index future market.

\section{Conclusion}

In this paper, our aim was to analyze how HFT influences exogenous liquidity risk of China's stock index future market. First, we make a problem statement about the concepts of exogenous liquidity risk, and then the experimental data are set according to the policy of trade restriction published in September 2015. To validate the relationship between HFT and liquidity risk, the VAR model is introduced to analyze the correlation between four agent indexes related to liquidity and HFT activeness. The experimental data have shown that HFT improves liquidity indicated by HFT causing fewer relative spread, effective spread, adverse selection, and larger realized spread.

Furthermore, the LVaR model is introduced to calculate how HFT influences the exogenous liquidity risk. The finding is that the exogenous liquidity risk drops from $16.89 \%$ after trade restriction to $4.04 \%$ before trade 
restriction, which in turn demonstrates HFT can reduce the exogenous liquidity risk level.

However, as the stock index future market and the spot market are highly related, the research about analyzing the impact of HFT on the spot market is a promising area. Therefore, for future research, it is necessary to study the cross-market HFT behaviour and the cross-market risk transmission mechanism to provide further theoretical and method guidance for the healthy and orderly development of China's stock index futures products and financial derivative markets.

\section{Data Availability}

Access to data used in this paper is restricted due to commercial confidentiality. Limited data can be provided upon request.

\section{Conflicts of Interest}

The authors claim no conflicts of interest.

\section{References}

[1] Y. Zhang and S. Ding, "Return and volatility co-movement in commodity futures markets: the effects of liquidity risk," Quantitative Finance, vol. 18, no. 9, pp. 1471-1486, 2018.

[2] P. Stoimenov, "Philippe jorion, value at risk, 3rd ed: the new benchmark for managing financial risk," Statistical Papers, vol. 52, no. 3, pp. 737-738, 2011.

[3] Y. Amihud and H. Mendelson, "Liquidity and asset prices: financial management implications," Financial Management FINAN MANAGE, vol. 17, p. 03, 1988.

[4] C. Lawrence and G. N. Robinson, Liquidity, dynamic hedgingand value at risk, vol. 63-72, Edition Risk Publications, London, UK, 2019.

[5] K. Dowd, Beyond Value at Risk: The New Science of Risk Management, John Wiley and Sons, New York, NY, USA, 1998.

[6] Ľ. Pástor and R. F. Stambaugh, "Liquidity risk and expected stock returns," Journal of Political Economy, vol. 111, no. 3, pp. 642-685, 2003.

[7] A. Bangia, F. X. Diebold, T. Schuermann, and J. Stroughair, Modeling Liquidity Risk With Implications for Traditional Market Risk Measurement and Management, Center for Financial Institutions Working Papers 99-06, Wharton School Center for Financial Institutions, University of Pennsylvania, Philadelphia, PA, USA, 1998.

[8] Z. Lu, A study on liquidity risk of open-ended fund and its management-an analysis based on china securities market during its transition, $\mathrm{PhD}$ thesis, Jinan University, Jinan, China, 2005.

[9] J. Danielsson, Financial Risk Forecasting: The Theory AndPractice Of Forecasting Market Risk With Implementation In $R$ And Matlab, vol. 588, John Wiley \& Sons, Hoboken, NY, USA, 2011.

[10] L. De Haan and Ana Ferreira, Extreme Value Theory: An Introduction, Springer Science \& Business Media, Berlin, Germany, 2007.

[11] R. B. Nelsen, An Introduction to Copulas, Springer Science \& Business Media, Berlin, Germany, 2007.
[12] Y. HisataY. Yamai et al., Research toward the Practical Application of Liquidity Risk Evaluation Methods, Institute for Monetary and Economic Studies, Bank of Japan, Tokyo, Japan, 2000.

[13] F. Hu and Q. Song, "Research on the market liquidity risk of China listed commercial banks-based on la-var model," Journal of Financial Development Research, vol. 9, 2016.

[14] X. Zhu, "Stock portfolio liquidity risk measurement model: construction and test," Chinese Journal of Management Science, vol. 15, no. 1, pp. 6-11, 2007.

[15] X. Xu, H. Wang, and Z. Zheng, "Liquidity risk measurement and analysis of China's new otc market," Contemporary Economic Research, vol. 10, no. 11, pp. 82-89, 2017.

[16] Mu Tong, He Yi, and J. Wen, "Intraday liquidity risk: progress and shortages in international regulation," Financial Theory and Practices, vol. 6, no. 8, pp. 94-100, 2017.

[17] H. A. Akaike, "New look at statistical model identification," IEEE Transactions on Automatic Control, vol. AC19, p. 716M723, 1974.

[18] G. Schwarz, "Estimating the dimension of a model," The Annals of Statistics, vol. 6, no. 2, pp. 461-464, 1978.

[19] S. Chen, "Research on portfolio liquidity risk measurement based on VaR model," PhD Thesis, Huazhong University of Science and Technology, Wuhan, China, 2005.

[20] E. P. George, G. M. J. Box, G. C. Reinsel, and M. L. Greta, Time Series Analysis: Forecasting and Control, John Wiley \& Sons, Hoboken, NY, USA, 2015. 